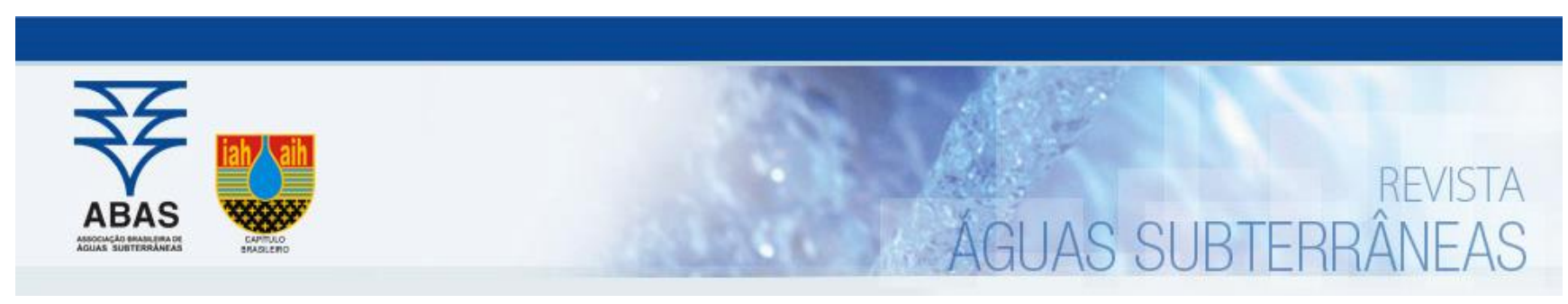

Artigos

\title{
Análise do potencial de lixiviação do inseticida sulfoxaflor em solos
}

\section{Analysis of the sulfoxaflor insecticide leaching potential in soils}

\section{Jonathas Gomes de Carvalho Marques; Suzana Maria Gico Lima Montenegro ${ }^{1}$ Marilia Regina Costa Castro Lyra2; Rogéria Mendes do Nascimento2; José Antônio Aleixo da Silva ${ }^{\circledR}$}

1 Universidade Federal de Pernambuco (UFPE), Recife, PE

2 Instituto Federal de Educação, Ciência e Tecnologia de Pernambuco (IFPE), Recife, PE

2 Universidade Federal Rural de Pernambuco (UFRPE), Recife, PE

$\triangle$ jonathasgomes@hotmail.com, suzanam.ufpe@gmail.com, mariliarcastro@uol.com.br, rogeriamendes@recife.ifpe.edu.br, jaaleixo@uol.com.br

\section{Palavras-chave:}

Águas subterrâneas.

Sulfoxaminas.

Groundwater Screening Index.
Contaminação ambiental.

\section{Resumo}

A utilização de agrotóxicos na agricultura é justificada pela necessidade de eliminação de diversas pragas, todavia, pode promover contaminação ambiental. Dentre esses produtos, está o princípio ativo sulfoxaflor, desenvolvido pela empresa Dow Agrosciences, atingindo grande sucesso no combate aos parasitas que se alimentam de seiva. Porém, tal composto é capaz de atingir negativamente espécies não-alvo, como formigas, minhocas e abelhas. Poucos estudos avaliaram a relação entre o sulfoxaflor e os recursos hídricos, especificamente os subterrâneos. Assim, a presente pesquisa visou analisar o potencial de tal agrotóxico em lixiviar no perfil do solo, atingindo os aquíferos. Para tanto, valeu-se de quatro modelos analíticos que, com parâmetros físico-químicos, fornecem output válido, que servem de base para estudos futuros mais aprofundados, são eles: GSI, LIX, RLPI e LEACH. Os quatro índices indicaram, cada um em seu espectro de valores, que o sulfoxaflor tem capacidade de lixiviar. GSI se destacou, pois alocou-o em classe de maior probabilidade de percolação. Ressalta-se, porém, que este estudo é preliminar, sugerindo-se análises mais robustas para que as conclusões a respeito dos impactos do uso e lixiviação do inseticida para os recursos hídricos subterrâneos sejam confirmadas.

Abstract

Keywords:

Pesticides use in agriculture is justified by the need of eliminating several pests, however, it allows environmental contamination. Among these products, the active ingredient sulfoxaflor, developed by the company Dow Agrosciences, achieving great success in combating parasites that are sap-feeding. However, the compound is capable of negatively affecting non-target species such as ants, earthworms, and bees. Few studies have evaluated the relationship between sulfoxaflor and water resources, specifically groundwater. Thus, this research aimed to analyze the pesticide leaching potential in the soil profile, reaching aquifers. For that, it used four analytical models that, with physical-chemical parameters, provide valid output, which serve as a basis for further in-depth studies, they are: GSI, LIX, RLPI and LEACH. The four indexes indicated, each in its range of values, that sulfoxaflor is capable of leaching. GSI stood out because it placed it in a class with a higher percolation probability. It should be noted, however, that this study is preliminary, suggesting more robust analyzes so that the conclusions regarding the use of the impacts of using this insecticide on groundwater resources are confirmed.

DOI: http:/dx.doi.org/10.14295/ras.v34i3.29921

\section{INTRODUÇÃO}

A agricultura convencional se mostra extremamente dependente da utilização de agrotóxicos para o combate às pragas. A tese inicialmente posta pelos defensores da Revolução Verde* era a de que sem esses produtos químicos haveria impossibilidade de abastecimento da população mundial.
Dentro desse espectro de compostos, encontra-se o inseticida sulfoxaflor (Figura 1) do grupo das sulfoxaminas, que tem como fórmula $\mathrm{C}_{10} \mathrm{H}_{10} \mathrm{~F}_{3} \mathrm{~N}_{3} \mathrm{OS}$, e é classificado, segundo critérios da Agência Nacional de Vigilância Sanitária (ANVISA), como Classe III (medianamente tóxico). Ele encontra-se apro vado para as seguintes culturas: algodão, arroz, citros, feijão, melão, melancia, milho, soja, tomate e trigo (ANVISA, 2019).

* Revolução Verde, ocorrida a partir da década de 1960, pode ser definida como o conjunto de modificações na agricultura convencional mundial, com destaque para a modificação genética e inserção de insumos químicos e tecnológicos visando ao aumento da produtividade agrícola. 
Figura 1 - Estrutura química do princípio ativo Sulfoxaflor

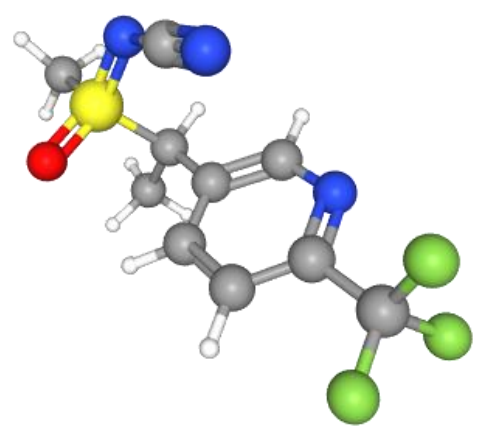

Fonte: Pubchem (2019).

[1-[6-(trifluoromethyl)pyridin-3-yl]ethyl]methyl(oxido)-4-sulfanylidenecyanamide apresenta bons resultados no controle de insetos que se alimentam de seiva (BABCOCK et al., 2010; WATSON et al., 2011; ZHU et al., 2011; XU et al, 2012; LONGHURST et al., 2013; SPARKS et al., 2013; NUGENT et al., 2015; BUYSSE et al., 2016; LOSO et al., 2016; WATSON et al., 2017; BACCI; CONVERTINI; ROSSARO, 2018; YANG et al., 2020), a exemplo dos pulgões (BABCOCK et al., 2010; WATSON et al., 2011; ZHU et al., 2011; XU et al, 2012; NUGENT et al., 2015; BUYSSE et al., 2016; LOSO et al., 2016; BACCI; CONVERTINI; ROSSARO, 2018), Lygus (BABCOCK et al., 2010; ZHU et al., 2011; XU et al, 2012; NUGENT et al., 2015; BUYSSE et al., 2016), funis (ZHU et al., 2011; XU et al, 2012; NUGENT et al., 2015; BUYSSE et al., 2016; LOSO et al., 2016) e moscas brancas (BABCOCK et al., 2010; ZHU et al., 2011; XU et al, 2012; LONGHURST et al., 2013; NUGENT et al., 2015; BUYSSE et al., 2016; LOSO et al., 2016; BACCI; CONVERTINI; ROSSARO, 2018).

Trata-se de um princípio ativo novo, desenvolvido e comercializado pela empresa Dow Agrosciences a partir de 2007 (ZEWAIN et al., 2013), e que já é um dos inseticidas mais usados nas culturas de arroz e trigo (TIAN et al., 2016). No Brasil, foi registrado no fim de 2018, gerando, em pouco tempo, a aprovação de nove marcas comerciais, a saber: Closer, Closer SC, Exor, Exor SC, Expedition, Haffor, Sortic, Verter, Verter SC, todos da referida empresa. Cerca de 33\% deles estão alocados na Classe I (extremamente tóxico) (AGROFIT, 2019).

Todavia, para saber se este composto apresenta riscos potenciais de contaminação ao meio ambiente, faz-se necessário a análise de seus efeitos em organismos em espécies não alvo (PAN; LU; WANG, 2017). Nesse sentido, sabe-se que concentrações subletais de sulfoxaflor em solenopsis invicta é capaz de impactá-los negativamente (PAN; LU; WANG, 2017). Este composto causa ainda efeitos danosos em abelhas, por gerar menos indivíduos férteis, o que impactará, em médio e longo prazo, sua quantidade na natureza e a realização das funções ecossistêmicas (CENTNER; BREWER; LEAL, 2018; SIVITER; BROWN; LEADBEATER, 2018; SIVITER et al., 2019; EFSA, 2020).

Além disso, há comprovações de que a exposição ao sulfoxaflor causa danos oxidativos em minhocas, alterações no metabolismo energético e ciclo de ureia, com possibilidade de al- teração do DNA (FANG et al., 2018). Dessa forma, pelo fato desse organismo servir de ponto de entrada do inseticida para a teia alimentar, deve se ter especial atenção sobre os riscos do sulfoxaflor ao meio ambiente (FANG et al., 2018; GHOSH et al., 2020; ZHANG et al., 2020). Sabe-se ainda que este princípio ativo estimula o aparecimento de tumores em fígado de ratos e camundongos (LEBARON et al., 2013; LEBARON et al., 2014; RASOULPOUR et al., 2014).

Ademais, possui potencial de bioacumulação na cadeia alimentar, sendo capaz de afetar, desta forma, a saúde humana (CHEN et al., 2016; TIAN et al., 2016), apesar de ser rapidamente biodegradado no solo. A preocupação é tanta que têm sido desenvolvidos novos métodos de detecção dos resíduos deste composto em alimentos variados como leite, carnes, ovos, hortaliças, dentre outros (XU et al., 2012; DO et al., 2013; KIM et al., 2016; TIAN et al., 2016; CHUANG et al., 2017; CUI et al., 2018; ZHANG et al., 2019).

A Agência de Meio Ambiente dos Estados Unidos da América (EUA) (USEPA) procedeu ao cancelamento dos registros do sulfoxaflor (em decisão no ano de 2015) baseado em dúvidas sobre a possibilidade de efeitos em populações de abelhas (EPA, 2015). Porém, liberou o inseticida novamente em 2019. Somente os seguintes países da União Europeia estão com registro ativo do agrotóxico para o inseticida: República Checa, Grécia, França, Irlanda e Eslováquia (LEWIS et al., 2016). As análises dos demais governos locais do bloco econômico demonstram e ratificam o princípio da precaução quanto a sua utilização.

Não obstante a evolução de análises e estudos nas matrizes ambientais citadas anteriormente, percebe-se a falta de pesquisas mais aprofundadas que associem a contaminação com a matriz recursos hídricos, principalmente por ter sido o composto desenvolvido recentemente, bem como o grupo químico em que está inserido. Essa falta de investigações, que associem o sulfoxaflor à contaminação hídrica, implica em aceite a um risco ainda desconhecido para a população que é abastecida com este recurso, principalmente em áreas em que ele é utilizado para o combate às pragas.

Assim, para tentar entender os meandros desta área de conhecimento, observa-se que inseticidas que se encontram no modo de ação do grupo 4 (moduladores competitivos de re- 
ceptores nicotínicos da acetilcolina) estabelecido pelo Comitê de Ação à Resistência de Inseticidas (IRAC, 2020), mesmo grupo das sulfoxaminas, já possuem estudos que relatam sua contaminação em águas subterrâneas, a exemplo de clotianidina (GOVERNMENT OF QUEBEC, 2011; BROWNE, 2017; WISCONSIN, 2018; ALFORD; KRUPKE, 2019; WISCONSIN, 2019), imidacloprido (NASCIMENTO, 2013; PITARCH et al., 2016; BROWNE, 2017; SCHATZ, 2017; WISCONSIN, 2018; WISCONSIN, 2019), tiametoxam (GOVERNMENT OF QUEBEC, 2011; BRADFORD; HUSETH; GROVES, 2018; WISCONSIN, 2018), dinotefuran (BRADFORD; GROVES, 2019), apesar de diferenças químicas e bioquímicas existentes dentro deste agrupamento (SPARKS et al., 2013).

Assim, para uma avaliação inicial deste risco, uma das ferramentas bastante utilizadas é a aplicação de índices (equações matemáticas) que prevejam o comportamento dos agrotóxicos no perfil do solo, por meio de seus parâmetros físico-químicos, que são de fácil obtenção (LASKOWSKI et al., 1982; BISHOP, 1986; HORNSBY, BUTTLER, BROWN, 1993; MARIOT et al., 2009; NASCIMENTO, 2013; BRITTO et al., 2015; GUERRA et al., 2016; MARQUES, 2017; SOARES; FARIA; ROSA, 2017; ESTEVES et al., 2018; ISMAEL; ROCHA, 2019; MARQUES et al., 2019; SILVA et al., 2019).

Dentre os autores citados, encontram-se estudos de validação/criação dos índices LIX (KOGAN et al., 2007; MARIOT et al., 2009; GUERRA et al., 2016), GSI (BISHOP, 1986), RLPI (HORNSBY, BUTTLER, BROWN, 1993), LEACH (LASKOWSKI et al., 1982; KOGAN et al., 2007). Os demais estudos aplicaram modelos (como GUS, RLPI, LIX, GSI, LEACH) em localidades distintas, a saber: sub bacia do Natuba (PE) (NASCIMENTO, 2013; MARQUES, 2017; MARQUES et al., 2019), baixo rio São
Francisco (BRITTO et al., 2015), Campo Novo do Parecis (MT) (SOARES; FARIA; ROSA, 2017), região metropolitana de João Pessoa (PB) (ISMAEL; ROCHA, 2019), perímetro irrigado Poção da Ribeira (ESTEVES et al., 2018), além de municípios de Casa Nova e Sento Sé (BA) (SILVA et al., 2019).

Frente ao exposto, o presente estudo tem como objetivo avaliar a potencialidade do sulfoxaflor de contaminar o meio ambiente, em especial o recurso hídrico subterrâneo, por meio do processo de lixiviação, de modo que represente o comportamento do composto no ambiente e, assim, sirva de embasamento para pesquisas futuras.

\section{METODOLOGIA}

Visando entender o comportamento ambiental do inseticida sulfoxaflor no recurso hídrico subterrâneo, utilizou-se alguns dos modelos analíticos verificados por Marques et al. (2019). Segundo os autores, os índices GSI, GUS, LEACH, LIX e RLPI tendem a fornecer informação similar no que se refere à capacidade de lixiviação dos agrotóxicos. Esta inferência pôde ser feita após padronização dos dados em relação à média e posterior análise de variância dos outputs (com p-valor 0,05), com distribuição da base de dados considerada normal pelo teste de Kolmogorov-Smirnov (LOPES; CASTELO BRANCO; SOARES, 2013).

Foram utilizados, então, quatro dos modelos analisados por Marques et al. (2019), a saber: Groundwater Screening Index - GSI (BISHOP, 1986), índice de LIX (SPADOTTO, 2002), Relative Leaching Potential Index - RLPI (HORNSBY, BUTTLER, BROWN, 1993) e Leaching Index - LEACH (LASKOWSKI et al., 1982) (Tabela 1).

Tabela 1 - Dados de entrada para os modelos matemáticos analíticos selecionados

\begin{tabular}{|c|c|c|c|}
\hline Parâmetros físico-químicos & Sigla & Unidade & Sulfoxaflor \\
\hline Coeficiente de adsorção ao carbono orgânico & $\mathrm{K}_{\mathrm{oc}}$ & $\mathrm{mL} / \mathrm{g}$ & 40,8 \\
\hline Meia vida no solo & $\mathrm{T}_{1 / 2}$ & dias & 2,2 \\
\hline Solubilidade em água $\left(20^{\circ} \mathrm{C}\right)$ & S & $\mathrm{mg} / \mathrm{L}$ & 568 \\
\hline Coeficiente de partição octanol-água (pH 7 a 20 $)$ & $\mathrm{K}_{\mathrm{ow}}$ & - & 6,34 \\
\hline Pressão de vapor $\left(20^{\circ} \mathrm{C}\right)$ & $V_{p}$ & $\mathrm{MPa}$ & $1,4 \times 10^{-3}$ \\
\hline
\end{tabular}

Analisando-se alguns dos parâmetros elencados, definese meia vida no solo ( $\left.\mathrm{T}_{1 / 2}\right)$ como o tempo (em dias) em que uma dada substância tem potencial de ser degradada à metade da quantidade que foi pulverizada. 0 coeficiente de partição octanol-água, ou Kow, por sua vez, é a medida da hidrofilicidade de um dado composto (OLIVEIRA, 2007) e o coeficiente de adsorção ao carbono orgânico (Koc), é um parâmetro que indica a capacidade de o composto adsorver ao solo (Tabela 2). 
Tabela 2 - Valores do coeficiente de adsorção ao carbono orgânico e sua classificação

\begin{tabular}{ccc}
\hline $\mathrm{K}_{\mathrm{oc}(\mathrm{mL} / \mathrm{g})}$ & Log Koc & Classificação \\
\hline$<10$ & $<1$ & Altamente móvel \\
$10-100$ & $1-2$ & Móvel \\
$100-1.000$ & $2-3$ & Moderadamente móvel \\
$1.000-10.000$ & $3-4$ & Ligeiramente móvel \\
$10.000-100.000$ & $4-5$ & Dificilmente móvel \\
$>100.000$ & $>5$ & Imóvel \\
\hline
\end{tabular}

Fonte: FAO (2000).

As fórmulas utilizadas nos modelos estão expostas abaixo:

$$
\begin{aligned}
& \mathrm{GSI}=\ln \left[\left(\mathrm{S} \cdot \mathrm{T}_{1 \backslash 2}\right) / \log \mathrm{K}_{\mathrm{ow}}\right] \\
& \mathrm{LIX}=\exp \left(-\mathrm{K} \cdot \mathrm{K}_{\mathrm{oc}}\right) \\
& \mathrm{RLPI}=10 *\left(\mathrm{~K}_{\mathrm{oc}} / \mathrm{T}_{1 / 2}\right) \\
& \mathrm{LEACH}=\left[\left(\mathrm{S} \cdot \mathrm{T}_{1 \backslash 2}\right) /\left(\mathrm{V}_{\mathrm{p}} \cdot \mathrm{K}_{\mathrm{oc}}\right)\right]
\end{aligned}
$$

Os modelos utilizados fazem uso, tão somente, das características físico-químicas, e produzem uma classificação inicial importante do potencial do produto percolar no perfil do solo, não o associando a nenhuma localidade agrícola (com seus dados climáticos, pedológicos, dentre outros), o que serve de base para estudos regionais mais aprofundados.

\section{RESULTADOS E DISCUSSÃO}

\subsection{Avaliação dos parâmetros físico-químicos do sulfoxa- flor}

Avalia-se que o sulfoxaflor possui baixa capacidade de sorver ao solo (com Koc de 40,8), sugerindo, em princípio, alta mobilidade. Por isso é capaz, segundo EPA (2019), de contaminar os recursos hídricos subterrâneos, principalmente quando está associado a intensas precipitações. Por sua vez, os cursos d'água superficiais podem ser atingidos devido à deriva no processo de pulverização do produto.
O sulfoxaflor apresenta baixa meia vida no solo (2,2 dias), o que indica que a contaminação só é possível quando associada a precipitações em curto espaço de tempo posterior à aplicação. A solubilidade (S) é outro parâmetro importante, pois revela a capacidade de o composto ser dissolvido na água (temperatura a $20^{\circ} \mathrm{C}$ ). 0 valor estimado para este agrotóxico é de $568 \mathrm{mg} / \mathrm{L}$, ao passo em que o valor limítrofe, considerado por Wilkerson e Kim (1986), para a indicação de alto potencial de solubilização em água é de $7 \mathrm{mg} / \mathrm{L}$. Ou seja, o composto é cerca de 81 vezes maior que o valor de referência.

Por sua vez, pressão de vapor $\left(V_{p}\right)$ é definida, por Atkins e Paula (2008), como a pressão que o composto exerce quando há equilíbrio dinâmico (entre os estados líquido e gasoso). Na qualidade de um indicador de volatilidade do composto, uma vez que este parâmetro para o sulfoxaflor

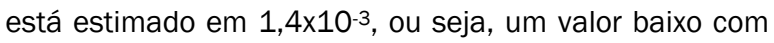
menor tendência de dissipação pelo ar, há indicativo de sua capacidade de lixiviar.

\begin{tabular}{|c|c|c|}
\hline $\begin{array}{c}\text { Modelos matemáticos } \\
\text { analíticos }\end{array}$ & Resultados & Valores de referência \\
\hline GSI & 7,35 & $\begin{array}{l}\text { GSI<1: Contaminação improvável } \\
1<\mathrm{GSI}<3 \text { : possível contaminação } \\
\text { GSI > 3: contaminação provável } \\
\underline{G S I}>\text { : }\end{array}$ \\
\hline LIX & $2,6 \times 10^{-6}$ & $\begin{array}{l}\text { LIX }=0 \text { não lixivia } \\
0<\operatorname{LIX}<0,1 \text { zona de transição } \\
\text { LIX } \geq 0,1 \text { lixivia }\end{array}$ \\
\hline RLPI & 185,45 & * \\
\hline LEACH $\left(\log _{10}\right)$ & 4,33 & * \\
\hline
\end{tabular}

\subsection{Estimativa do potencial de lixiviação do sulfoxaflor}

Nesta seção são apresentadas as estimativas de lixiviação do sulfoxaflor, de acordo com cada modelo (Tabela 3).
O índice GSI demonstrou que o composto deve ser tratado com especial atenção, ou seja, está inserido na faixa dos compostos com maior capacidade de lixiviar, dentro de sua escala, e possui a necessidade de atenção quando de sua aplicação, pulverizando-o somente sob condições pedológicas, de manejo e de precipitação que retardem o processo de percolação. O LIX alocou-o em classe de transição, principalmente pelo fato de o coeficiente de adsor- 
ção ao carbono ser baixo, o que sugere uma possível mobilidade do composto no perfil do solo.

Todavia, apesar de demonstrar outputs similares ao GSI e demais modelos, LIX não apresentou resultado tão elevado quanto o índice anterior, possivelmente por não incluir em seu cálculo a solubilidade, parâmetro que demonstra a elevada capacidade de ser dissolvida em água, tão somente o $\mathrm{K}_{\mathrm{oc}}$.

Quando se analisou os índices LEACH e RLPI, efetuou-se comparações com dez agrotóxicos considerados com baixo potencial de lixiviar (e que obtiveram valor para o LEACH), indicados no estudo de Marques et al. (2019). Observou-se uma variação de -4,68 até -1,32 entre os valores destes compostos contrastados no modelo LEACH. Além disso, como forma de gerar melhores comparativos, foram analisados também os inseticidas clotianidina, imidacloprido, tiametoxam e dinotefuran que são classificados no grupo 4 (moduladores competitivos de receptores nicotínicos da acetilcolina) do Comitê de Ação à Resistência de Inseticidas (Tabela 4).

$\underline{\text { Tabela } 4 \text { - Comparação entre sulfoxaflor e outros princípios ativos, com base no índice LEACH }}$

\begin{tabular}{cccccc} 
Ingredientes ativos & koc & T1/2 & S & Vp & LEACH (Log10) \\
\hline Sulfoxaflor & 40,8 & 2,2 & 568 & $1,4 \times 10^{-3}$ & 4,34 \\
Clotianidina & 123 & 545 & 340 & $2,8 \times 10^{-8}$ & 10,73 \\
Imseticidas do grupo de ação 4 do IRAC & 9,04 \\
Tiametoxam & 262 & 191 & 610 & $4 \times 10^{-7}$ & 9,74 \\
Dinotefuran & 26 & 82 & 39830 & $1,7 \times 10^{-3}$ & 7,86 \\
Princípios ativos de agrotóxicos do estudo de Marques et al. (2019) \\
Mancozebe & 998 & 0,1 & 6,2 & $1,3 \times 10^{-2}$ & $-1,32$ \\
Trifloxixtrobina & 2377 & 0,34 & $6,1 \times 10^{-1}$ & $3,4 \times 10^{-3}$ & $-1,59$ \\
Lambda-Cialotrina & 283707 & 175 & $5 \times 10^{-3}$ & $2 \times 10^{-4}$ & $-1,81$ \\
Cipermetrina & 156250 & 60 & $9 \times 10^{-3}$ & $2,3 \times 10^{-4}$ & $-1,82$ \\
Alfacipermetrina & 57889 & 35 & $4 \times 10^{-3}$ & $3,4 \times 10^{-4}$ & $-2,14$ \\
Malathion & 1800 & 0,17 & 148 & 3,1 & $-2,34$ \\
Beta Ciflutrina & 64300 & 13 & $1,2 \times 10^{-3}$ & $5,6 \times 10^{-5}$ & $-2,36$ \\
Espiromesifeno & 30900 & 4,1 & $1,3 \times 10^{-1}$ & $7 \times 10^{-3}$ & $-2,60$ \\
Fluazifop-P-Butil & 3394 & 1 & $9,3 \times 10^{-1}$ & $1,2 \times 10^{-1}$ & $-2,64$ \\
Deltametrina & 10240000 & 13 & $2 \times 10^{-4}$ & $1,24 \times 10^{-5}$ & $-4,68$ \\
\hline Fonte: Pesticides Properties Database & $($ PPDB) & $($ LEWIS et al., 2016$) ;$ Pesticide Action Network
\end{tabular}

(PAN) (KEGLEY et al., 2016).

O sulfoxaflor obteve o valor de LEACH ( $\left.\log _{10}\right)$ estimado em 4,33 , bem acima do agrotóxico que mais pontuou dentre o grupo de compostos extraídos de Marques et al., (2019) $(-1,59)$. Todavia, ficou com valor abaixo, quando comparado com os agrotóxicos (inseticidas) do mesmo grupo de ação. Isto indica que ele apresenta risco de lixiviar, entretanto, em menor grau quando analisado a outros inseticidas com características semelhantes.

Por sua vez, ao analisar o RLPI nas mesmas condições e requisitos do modelo anterior, obteve-se uma variação entre 7208 a 787.692 nos princípios ativos contrapostos, do estudo de Marques et al. (2019), e de 82 a 545, nos inseticidas do grupo 4 do IRAC. Por sua vez, o sulfoxaflor apresentou valor de 185,45 (Tabela 5).

Ressalta-se, que ao contrário dos outros modelos, o RLPI deve ser entendido por meio do raciocínio de que quanto maior o valor obtido, menor é o potencial de lixiviar (OLIVEIRA, 2007; NASCIMENTO, 2013). Dessa forma, os modelos LEACH e RLPI apontam que o sulfoxaflor deve ser avaliado como potencial lixiviante, confirmado pelos índices GSI e LIX. Todavia, os resultados indicam que ele tem menor tendência de lixiviar quando confrontado com os inseticidas do mesmo grupo de ação.

As pequenas variações entre os índices supracitados são possibilidades possiveis quando se trabalha com aplicação de índices, uma vez que há incertezas envolvidas, principalmente por não serem incluídas todas as variáveis e relações existentes no processo ambiental analisado. Por isso, na qualidade de estudos iniciais, devem ser validados por pesquisas com métodos mais robustos. 
Tabela 5 - Comparação entre sulfoxaflor e outros agrotóxicos, com base no índice LEACH

\begin{tabular}{cccc} 
Ingredientes ativos & Koc & T1/2 & RLPI \\
\hline Sulfoxaflor & 40,8 & 2,2 & 185,45 \\
Inseticidas do grupo de ação 4 do IRAC \\
Clotianidina & 123 & 545 & 2,25 \\
Imidacloprido & 262 & 191 & 13,71 \\
Tiametoxam & 56,2 & 50 & 11,24 \\
Dinotefuran & 26 & 82 & 3,17 \\
Princípios ativos de agrotóxicos do estudo de Marques et al. (2019) \\
Mancozebe & 998 & $1 \times 10^{-1}$ & 99800 \\
Trifloxixtrobina & 2377 & $3,4 \times 10^{-1}$ & 69911,76 \\
Lambda-Cialotrina & 283707 & 175 & 16211,82 \\
Cipermetrina & 156250 & 60 & 26041,66 \\
Alfacipermetrina & 57889 & 35 & 16539,71 \\
Malathion & 1800 & $1,7 \times 10^{-1}$ & 105882,35 \\
Beta Ciflutrina & 64300 & 13 & 49461,53 \\
Espiromesifeno & 30900 & 4,1 & 75365,85 \\
Fluazifop-P-Butil & 3394 & 1 & 33940 \\
Deltametrina & 10240000 & 13 & 7876923,07 \\
\hline
\end{tabular}

Fonte: Pesticides Properties Database (PPDB) (LEWIS et al., 2016); Pesticide Action Network (PAN) (KEGLEY et al., 2016).

Todavia, são ótimos indicadores para uma análise inicial sobre os riscos de contaminação de determinado princípio ativo no meio ambiente (MARQUES, 2017). Isto acontece mesmo tendo as equações utilizados alguns parâmetros em comum, pois, em sua maioria, a metodologia utilizada para estimar a potencialidade de lixiviação de cada agrotóxico é distinta.

Dessa forma, é possível afirmar que o inseticida sulfoxaflor tem provável capacidade de lixiviar. Esta inferência é plausivel a partir dos resultados de GSI e LIX que indicam alta capacidade de lixiviação e provável lixiviação (zona de transição), respectivamente, coadunando-se com os indicativos das comparações na análise dos índices LEACH e RLPI que demonstram esse posicionamento do composto em uma zona transicional.

O fato de os índices GSI, LEACH e RLPI sugerirem provável lixiviação, e LIX zona de transição, deve servir como alerta para que os atores sociais pertinentes deem atenção ao sulfoxaflor no que se refere a monitoramento ambiental. Oliveira (2007) vai além e propõe que essas informações embasem e sirvam de critério, juntamente com outros dados como custo e eficiência agrícola, para a prescrição deste produto para os agricultores.

Assim, alerta-se para que a utilização desse agrotóxico no meio ambiente seja de forma racional, tendo em vista seu potencial contaminante. Sugere-se que este seja alvo de análises mais profundas para que seu uso na agricultura se dê de uma forma mais sustentável, mitigando os impactos ambientais negativos, em especial no recurso hídrico subterrâneo.

\section{CONCLUSÕES}

Percebe-se que o inseticida sulfoxaflor, quando analisado quanto à capacidade de contaminação dos recursos hídricos subterrâneos, sugere alta probabilidade de lixiviação no perfil do solo, com recomendações de cautela no uso desse inseticida. Tendo em vista sua alta capacidade de solubilização é importante que, quando utilizado, seja aplicado em período de baixa pluviosidade, visando diminuir os riscos de percolação e, consequentemente, contaminação hídrica subterrânea.

Dentre os quatro índices analisados, GSI se destacou quanto aos resultados, pois alocou o composto na classe de maior probabilidade de contaminação, já o índice de LIX incluiu-o na classe de transição. Por sua vez, LEACH e RLPI, pelo fato de não possuírem estratificação própria, ao serem comparados com outros agrotóxicos, demonstram provável capacidade de lixiviação (zona transicional).

Sugere-se outras pesquisas que analisem de forma mais aprofundada o sulfoxaflor quanto ao seu risco de contaminar a água subterrânea, sendo realizado por meio de monitoramento contínuo, visando maior acurácia no embasamento para tomada de decisões no que se refere à utilização do composto. 


\section{REFERÊNCIAS}

AGROFIT. Consulta de ingrediente ativo: sulfoxaflor. Brasília: Ministério da Agricultura, Pecuária e Abastecimento. Disponivel em: http://agrofit.agricultura.gov.br/agrofit_cons/principal_agrofit_cons. Acesso em: 1 nov. 2019.

ALFORD, A. M.; KRUPKE, C. H. Movement of the neonicotinoid seed treatment clothianidin into groundwater, aquatic plants, and insetct herbivores. Environmental Science and Technology, v. 53, n. 24, p. 14368-14376, 2019. https://doi.org/10.1021/acs.est.9b05025

ANVISA. Sulfoxaflor. Brasília: Ministério da Saúde. Disponível em: http://portal.anvisa.gov.br/documents/111215/117782/S19+-+SULFOXA-

FLOR/86214cb1-380f-436f-a73b-d6ed9a811cd0. Acesso em: 01 nov. 2019.

ATKINS, P.; PAULA, J. Físico-química. 8. ed. São Paulo: Saraiva, 2008. Vol 1.

BABCOCK, J. M.; GERWICK, C. B.; HUANG, J. X.; LOSO, M R.; NAKAMURA, G.; NOLTING, S. P.; ROGERS, R. B.; SPARKS, T. C.; THOMAS, J.; WATSON, G. B.; ZHU, Y. Biolog ical characterization of sulfoxaflor, a novel insecticide. Pest Management Science, v. 67, n. 3, p. 328-334, 2010.

BACCI, L.; CONVERTINI, S.; ROSSARO, B. A review of sulfoxaflor, a derivative of biological acting substances as a class of insecticides with a broad range of action against many insect pests. Journal of Entomological and Acarological Research, v. 50, n. 3, p. 51-71, 2018. https://doi.org/10.4081/jear.2018.7836

BISHOP, K. C. Industry's perspective on agricultural chemicals in water supply and drainage. In: PROCEEDINGS "TOXIC SUBSTANCES IN AGRICULTURAL WATER SUPPLY AND DRAINAGE". 1986. Washington. [Anais...]. U.S Comitee of irrigation and drainage: Washington: FAO, 1986.

BRADFORD, B. Z.; HUSETH, A. S.; GROVES, R. L. Widespread detections of neonicotinoid contaminants in central Wisconsin groundwater. PLOS ONE, v. 13, n. 10, p. 1-17, 2018. https://doi.org/10.1371/journal.pone.0201753

BRADFORD, B. Z.; GROVES, R. L. An overview of neonicotinoid in insecticide contaminants in central Wisconsin's surface and groundwater systems. In: PROCEEDINGS OF THE 2019 WISCONSIN AGRIBUSINESS CLASSIC. 2019. [Anais...]. Wisconsin: University of Wisconsin Extension, 2019.

BRITTO, F. B.; SILVA, T. M. M.; VASCO, A. M.; AGUIAR NETTO, A. O.; CARVALHO C. M. Avaliação do risco de contaminação hídrica por agrotóxicos no perímetro irrigado betume no baixo rio São Francisco. Revista Brasileira de Agricultura Irrigada, v. 9, n. 3, p. 158-170, 2015 https://doi.org/10.7127/rbai.v9n300301

BROWNE, D. C. Neonicotinoids in groundwater: presence and fate in two distinct hydrogeologic settings in Ontario, Canada. 2017. 227 f. Dissertação (Mestrado em Ciência Aplicada em Engenharia) - University of Guelph, 2017.
BUYSSE, A. M.; NUGENT, B. M.; WANG, N. X.; BENKO, Z.; BREAUX, N.; ROGERS, R.; ZHU, Y. Studies toward under standing the SAR around the sulfoximine moiety of thesapfeeding insecticide sulfoxaflor. Pest Management Science, v. $73, \quad$ n. $4, \quad$ p. $731-742,2016$. https://doi.org/10.1002/ps.4351

CENTNER, T. J.; BREWER, B.; LEAL, I. Reducing damages from sulfoxaflor use through mitigation measures to increase the protection of pollinator species. Land Use Policy, v. 75, p. 70-76, 2018. https://doi.org/10.1016/j.landusepol.2018.03.016

CHEN, Z.; DONG, F.; PAN, X.; XU, J.; LIU, X.; WU, X.; ZHENG, $Y$. Influence of Uptake Pathways on the Stereoselective Dissipation of Chiral Neonicotinoid Sulfoxaflor in Greenhouse Vegetables. Journal of Agricultural and Food Chemistry, v. 64, n. 13, p. 2655-2660, 2016. https://doi.org/10.1021/acs.jafc.5b05940

CHUANG, H. S.; EL-ATY, A. M. A.; KIM, S. W.; LEE, H. S.; RAHMAN, M. M.; KABIR, M. H.; SHIN, H. C.; SHIM, J. H. Simultaneous determination of sulfoxaflor and its metabolites, X11719474 and X11721061, in brown rice and rice straw after field application using LC-MS/MS. International Journal of Environmental Analytical Chemistry, v. 97, n. 2, p. 99-111, 2017. https://doi.org/10.1080/03067319.2017.1282473

CUI, S. H.; LI, Z. Y.; CHENG, G.; LI, R. J.; WANG, Y.; ZHANG, X. Y.; ZHAO, F. Determination of sulfoxaflor, pyrifluquinazon and spirotetramat residues in fruits and vegetables by UPLC-MS/MS. China Food Plubishing Co, p. 1-10, 2018.

DO, J. A.; LEE, M. Y.; PARK, H.; KWON, J. E.; JANG, H.; CHO, Y. J.; JANG, I. H.; LEE, S. M.; CHANG, M. I.; OH, J. H.; HWANG, I. G. Development and Validation of an Analytical Method for the Insecticide Sulfoxaflor in Agricultural Commodities using HPLC-UVD. Korean Journal of Food Science and Technology, v. 45, n. 2, p. 148-155, 2013. https://doi.org/10.9721/KJFST.2013.45.2.148

EFSA. Peer review of the pesticide risk assessment for the active substance sulfoxaflor in light of confirmatory data submitted. EFSA Journal, v. 18, n. 3, p. 1-15, 2020

EPA. Sulfoxaflor - Final Cancellation Order. [2015]. Washington: USEPA. Disponivel em: https://www.epa.gov/sites/production/files/2015-11/documents/final_cancellation_order-sulfoxaflor.pdf. Acesso em: 01 nov. 2019.

EPA. Ecological Risk Assessment for the Registration of Sulfoxaflor. [2019]. Washington: USEPA. Disponivel em: https://www.indianaenvironmentalreporter.org/content/posts/20190717-epa-approves-expanded-use-of-indiana-made-insecticide-toxic-to-bees/toxic.pdf. Acesso em 15 abr 2020

ESTEVES, C. S.; VASCO, A. N.; SANTOS, H. B.; SILVA, C. L.; ESTEVES, C. S. Riscos ambientais do uso de agrotóxicos na produção de hortaliças no município de Itabaiana, Sergipe. Revista Craibeiras de Agroecologia, v. 1, n. 1, p. 1-7, 2018. 
FANG, S.; ZHANG, Y.; YOU, X.; SUN, P.; QIU, J.; KONG, F. Lethal Toxicity and Sublethal Metabolic Interference Effects of Sulfoxaflor on the Earthworm (Eisenia fetida). Journal of Agricultural and Food Chemistry, v. 66, n. 45, p. $11902-$ 11908, https://doi.org/10.1021/acs.jafc.8b04633

FAO. Parameters of pesticides that influence processes in soil. [2000]. Disponivel em: http://www.fao.org/3/X2570E/X2570E06.htm. Acesso em: 30 jun 2020.

GOHSH, S.; OJHA, P. K.; CARNESECCHI, E.; LOMBARDO, A.; ROY K.; BENFENATI, E. Exploring QSAR modeling of toxicity of chemicals on earthworm. Ecotoxixology and Environmental Safety, v. 190, p. 1-9, 2020. https://doi.org/10.1016/i.ecoenv.2019.110067

GOVERNMENT OF QUEBEC. Pesticides et nitrates dans l'eau souterraine près de cultures de pommes de terre. Quebec: Ministère du Développement durable, de l'Environnement et des Parcs, Direction du suivi de l'état de l'environnement, Centre d'expertise en analyse environnementale du Québec, 2011. 54 p.

GUERRA, N.; OLIVEIRA JÚNIOR, R. S.; CONSTANTIN, J.; OLIVEIRA NETO, A. M.; GEMELLI, T. M. C. J.; GUERRA, A. Potencial de lixiviação de herbicidas utilizados na cultura da cana-de-açúcar. Revista Campo Digit@l, v. 11, n. 1, p. 4253, 2016.

HORNSBY, A. G.; BUTTLER, T. M.; BROWN, R. B. Managing pesticides for crop production and water quality protection: practical grower guides. Agriculture, Ecosystems \& Environment, v. 46, n. 1-4, p. 187-196, 1993. https://doi.org/10.1016/0167-8809(93)90023-I

IRAC. Modo de ação de inseticidas e acaricidas. Disponíve em: https://www.irac-br.org/modo-de-acao. Acesso em: 02 jul 2020.

ISMAEL, L. L.; ROCHA, E. M. R. Estimativa de contaminação de águas subterrâneas e superficiais por agrotóxicos em área sucroalcooleira, Santa Rita/PB, Brasil. Ciência \& Saúde Coletiva, v. 24, n. 12, p. 4665-4675, 2019. https://doi.org/10.1590/1413812320182412.27762017

KOGAN, M.; ROJAS, S.; GÓMEZ, P.; SUÁREZ, F.; MUÑOZ, J. F.; ALISTER, C. Evaluation of six pesticides leaching indexes using field data of herbicide application in Casablanca Valley, Chile. Water Science \& Technology, v. 56, n. 2, p. 169-178, 2007. https://doi.org/10.2166/wst.2007.486

KEGLEY, S. E.; HILL, B. R.; ORME, S.; CHOI, A. H. PAN Pesticide Database, Pesticide Action Network, North America (Oakland, CA, 2016). Disponivel em: http://www.pesticideinfo.org/Search_Chemicals.jsp. Acesso em: 04 out. 2017.

KIM, S. W.; RAHMAN, M. M.; EL-ATY, A. M. A.; KABIR, M. H.; NA, T. W.; CHOI, J. H.; SHIN, H. C.; SHIM, J. H. Simultaneous detection of sulfoxaflor and its metabolites, X11719474 and X11721061, in lettuce using a modified QuEChERS extraction method and liquid chromatography-tandem mass spectrometry. Biomedical Chromatography, v. 31, n. 6, p. 1-9, 2016. https://doi.org/10.1002/bmc.3885
LASKOWSKI, D. A.; GORING, C. A.; MCCALL, P. J.; SWANN, P. J. Terrestrial Environment. In: CONWAY, R. A. (Ed.). Environmental Risk Analysis for Chemicals. Nova lorque: Krieger Publishing Company, p. 198-240, 1982.

LEBARON, M. J.; GETER, D. R.; RASOULPOUR, R. J.; GOLLAPUDI, B. B.; THOMAS, J.; MURRAY, J.; KAN, H. L.; WOOD, A. J.; ELCOMBE, C.; VARDY, A.; MCEWAN, J.; TERRY, C.; BILLINGTON, R. An integrated approach for prospectively investigating a mode-of-action for rodent liver effects. Toxicology and Applied Pharmacology, v. 270, n. 2, p. 164173, 2013. https://doi.org/10.1016/i.taap.2013.04.009

LEBARON, M. J.; GOLLAPUDI, B. B.; TERRY, C.; BILLINGTON, R.; RASOULPOUR, R. J. Human relevance framework for rod ent liver tumors induced by the insecticide sul foxaflor. Critical Reviews in Toxicology, v. 44, n. 3, p. 1524 ,

2014.

https://doi.org/10.3109/10408444.2014.910751

LEWIS, K. A.; TZILIVAKIS, J.; WARNER, D. J.; GREEN, A. An international database for pesticide risk assessments and management. Human and Ecological Risk Assessment: An International Journal, v. 22, n. 4, p. 1050-1064, 2016. https://doi.org/10.1080/10807039.2015.1133242

LOPES, M. M.; CASTELO BRANCO, V. T. F.; SOARES, J. B. Utilização dos testes estatísticos de Kolmogorov-Smirnov e Shapiro-Wilk para verificação da normalidade para materiais de pavimentação. Transportes, v. 21, n. 1, p. 59-66, 2013. https://doi.org/10.4237/transportes.v21i1.566

LONGHURST, C.; BABCOCK, J. M.; DENHOLM, I.; GORMAN, K.; THOMAS, J. D.; SPARKS, T. C. Cross-resistance relationships of the sulfoximine insecticide sulfoxaflor with neonicotinoids and other insecticides in the whiteflies Bemisia tabaci and Trialeurodes vaporariorum. Pest Managemente Science, v. 69, n. 7, p. 809-813, 2013. https://doi.org/10.1002/ps.3439

LOSO, M. R.; BENKO, Z.; BUYSSE, A.; JOHNSON, T. C.; NUGENT, B. M.; ROGERS, R. B.; SPARKS, T. C.; WANG, N. X.; WATSON, G. B.; ZHU Y. SAR studies directed toward the pyridine moiety of the sap-feeding insecticide sulfoxaflor (Isoclast ${ }^{\mathrm{TM}}$ active). Bioorganic \& Medicinal Chemistry, v. 24, n. 3, p. 378-382, 2016. https://doi.org/10.1016/i.bmc.2015.11.022

MARIOT, E.; WEBER, O. L. S.; SPADOTTO, C. A.; DORES, E. F. G. C. Uso do índice LIX na previsão de lixiviação dos pesticidas metolacloro, endossulfam, clorpirifós, carbendazim e diurom. Pesticidas: Revista de Ecotoxicologia e Meio Ambiente, v. 19, p. 57-64, 2009. https://doi.org/10.5380/pes.v19i0.16555

MARQUES, J. G. C. Proposições para o gerenciamento do uso de agrotóxicos utilizados na bacia hidrográfica do Natuba, Vitória de Santo Antão, Pernambuco. 2017. 125 f. Dissertação (Mestrado Profissional em Gestão Ambiental) - Programa de Pós-Graduação em Gestão Ambiental, Instituto Federal de Educação, Ciência e Tecnologia de Pernambuco, Recife, 2017.

MARQUES, J. G. C.; LYRA, M. R. C. C.; CARVALHO, R. M. C. O.; NASCIMENTO, R. M.; SILVA, J. A. A.; MONTENEGRO, S. M. G. L. Comparação entre índices de potencial de lixiviação para agrotóxicos utilizados na Sub-Bacia do Natuba, 
Vitória de Santo Antão-Pernambuco. Águas Subterrâneas, v. $33, \quad$ n. $1, \quad$ p. $58-67,2019$ https://doi.org/10.14295/ras.v33i1.29239

NASCIMENTO, R. M. Impactos dos agrotóxicos na contaminação ambiental da produção de hortaliças no baixo rio Natuba, Pernambuco. 2013. 167 f. Tese (Doutorado em Engenharia Civil) - Centro de Tecnologia e Geociências, Universidade Federal de Pernambuco, Recife, 2013.

NUGENT, B. M.; BUYSSE, A. M.; LOSO, M. R.; BABCOCK, J. M.; JOHNSON, T. C.; OLIVER, M. P.; MARTIN, T. P.; OBER, M. S.; BREAUX, N.; ROBINSON, A.; ADELFINSKAYA, Y. Expanding the structure-activity relationship of sulfoxaflor: the synthesis and biological activity of $\mathrm{N}$-heterocyclic sulfoximines. Pest Management Science, v. 71, n. 7, p. 928936, 2015. https://doi.org/10.1002/ps.3865

OLIVEIRA, E. S. Indicadores geoambientais de qualidade das águas na Bacia do Córrego Sujo, Médio Vale do rio Paraíba do Sul, Teresópolis (RJ). 2007. 185 f. Tese (Doutorado em Geociências) - Pós-Graduação em Geociências, Universidade Federal Fluminense, 2007.

PAN, F.; LU, Y.; WANG, L. Toxicity and sublethal effects of sulfoxaflor on the red imported fire ant, Solenopsis invicta. Ecotoxicology and Environmental Safety, v. 139, p. 377 383, 2017. https://doi.org/10.1016/i.ecoenv.2017.02.014

PITARCH, E.; CERVERA, M. I.; PORTOLÉS, T.; IBÁÑNEZ, M.; BARREDA, M.; RENAU-PRUÑONOSA, A.; MORELL, I.; LÓPEZ, F.; ALBARRÁN, F.; HERNÁNDEZ, F. Comprehensive monitoring of organic micro-pollutants in surface and groundwater in the surrounding of a solid-waste treatment plant of Castellón, Spain. Science of the Total Environment, v. 548-549, p. 211-220, 2016 . https://doi.org/10.1016/j.scitotenv.2015.12.166

PUBCHEM. Sulfoxaflor. Disponível em: https://pubchem.ncbi.nlm.nih.gov/compound/Sulfoxaflor\#section=3D-Conformer. Acesso em: 25 out. 2019.

RASOULPOUR, R. J.; TERRY, C.; LEBARON, M. J.; STEBBINS, K.; ELLIS-HUTCHINGS, R.; BILLINGTON, R. Mode-of-action and human relevance framework analysis for rat Leydig cell tumors associated with sulfoxaflor. Critical Reviews in Toxicology, v. 44, n. 2, p. 25-44, 2014. https://doi.org/10.3109/10408444.2014.910750

SCHATZ, A. L. Accounting for concentrated and área sources of imidacloprid in groundwater, Suffolk County, Long Island, NY. 2017. 178 f. Dissertação (Mestrado em Engenharia Agrícola e Biológica) - Faculty of the Graduate School of Cornell University, Cornell University, 2017.

SILVA, P. T. S.; MEDEIROS, L. R. A.; CUNHA, T. J. F.; COSTA, N. D.; SALVIANO, A. M. Risco de contaminação das águas superficiais e subterrâneas por agrotóxicos em cultivos de cebola no entorno do lago de Sobradinho, estado da Bahia. Petrolina: Embrapa Semiárido, 2019. 26 p.

SIVITER, H.; BROWN, M. J. F.; LEADBEATER, E. Sulfoxaflor exposure reduces bumblebee reproductive success. Nature, v. 561, n. 7721, p. 109-112, 2018. https://doi.org/10.1038/s41586-018-0430-6
SIVITER, H.; HORNER, J.; BROWN, M. J. F.; LEADBEATER, E. Sulfoxaflor exposure reduces egg laying in bumblebees Bombus terrestris. Journal of Applied Ecology, v. 57, n. 1, p. 160-169, 2019. https://doi.org/10.1111/1365$\underline{2664.13519}$

SOARES, D. F.; FARIA, A. M.; ROSA, A. H. Análise de risco de contaminação de águas subterrâneas por resíduos de agrotóxicos no município de Campo Novo do Parecis (MT), Brasil. Engenharia Sanitária e Ambiental, v. 22, n. 2, p. 277-284, 2017. https://doi.org/10.1590/s1413$\underline{41522016139118}$

SPADOTTO, C. A. SCREENING METHOD FOR ASSESSING PESTICIDE LEACHING POTENTIAL. Pesticidas: Revista de Ecotoxicologia e Meio Ambiente, v. 12, p. 69-78, 2002. https://doi.org/10.5380/pes.v12i0.3151

SPARKS, T. C.; WATSON, G. B.; LOSO, M. R.; GENG, C.; BABCOCK, J. M.; THOMAS, J. D. Sulfoxaflor and the sulfoximine insecticides: chemistry, mode of action and basis for efficacy on resistant insects. Pesticide Biochemistry and Phisiology, v. 107, n. 1, p. 1-7, 2013. https://doi.org/10.1016/j.pestbp.2013.05.014

TIAN, C.; XU, J.; DONG, F.; LIU, X.; WU, X.; ZHAO, H.; JU, C.; WEI, D.; ZHENG, Y. Determination of Sulfoxaflor in Animal Origin Foods Using Dispersive Solid-Phase Extraction and Multiplug Filtration Cleanup Method Based on Multiwalled Carbon Nanotubes by Ultraperformance Liquid Chromatography/Tandem Mass Spectrometry. Journal of Agricultural and Food Chemistry, v. 64, n. 12, p. 2641-2646, 2016. https://doi.org/10.1021/acs.jafc.6b00285

WATSON, G. B.; LOSO, M. R.; BABCOCK, J. M.; HASLER, J. M.; LETHERER, T. J.; YOUNG, C. D.; ZHU, Y.; CASIDA, J. E.; SPARKS, T. C. Novel nicotinic action of the sulfoximine insecticide sulfoxaflor. Insect Biochemistry and Molecular Biology, v. 41, n. 7, p. 432-439, 2011. https://doi.org/10.1016/j.ibmb.2011.01.009

WATSON, G. B.; OLSON, M. B.; BEAVERS, K. W.; LOSO, M. R.; SPARKS, T. C. Characterization of a nicotinic acetylcholine receptor binding site for sulfoxaflor, a new sulfoximine insecticide for the control of sap-feeding insect pests. Pesticide Biochemistry and Phisiology, v. 143, p. 90-94, 2017. https://doi.org/10.1016/i.pestbp.2017.09.003

WILKERSON, M. R.; KIM, K. D. The pesticide contamination prevention act: setting specific numeral values. California: California Departament of Food and Agriculture - CDFA, 1986.

WISCONSIN. Bureau of Agrichemical Management Annual Report. Wisconsin: Wisconsin Department of Agriculture, Trade and Consumer Protection, 2018. 27 p.

WISCONSIN. A summary of groundwater and surface water test results for neonicotinoid insecticides from 2008 through 2016. Wisconsin: Wisconsin Department of Agriculture, Trade and Consumer Protection, 2019. 49 p.

XU, J.; DONG, F.; LIU, X.; LI, J.; LI, Y.; SHAN, W.; ZHENG, Y. Determination of sulfoxaflor residues in vegetables, fruits and soil using ultra-performance liquid chromatography/tandem mass spectrometry. Analytical Methods, n. 12, p. 4019-4024, 2012. https://doi.org/10.1039/c2ay25782c 
YANG, W. L.; DAI, Z. L.; CHENG, X.; GUO, L.; FAN, Z. X.; GE, F.; DAl, Y. J. Sulfoxaflor Degraded by Aminobacter sp. CGMCC 1.17253 through Hydration Pathway Mediated by Nitrile Hydratase. Journal of Agricultural and Food Chemistry, v. $68, \quad$ n. $16, \quad$ p. $4579-4587,2020$. https://doi.org/10.1021/acs.jafc.9b06668

ZEWAIN, Q. K.; KHALIL, H. K.; HASSAN, K. A.; AHMED, N. M. Evaluation of sulfoxaflor as a novel group insecticide against whitefly (bemisia tabaci genn.) (aleyrodidae: homoptera) on cucumber at nineveh governorate/kwer district. Diyala Agricultural Sciences Journal, v. 5, n. 1, p. 17 25, 2013.

ZHANG, X.; LI, T.; ZHANG, L.; HU, T.; FU, Y.; GUO, Z. Simultaneous determination of sulfoxaflor in 14 daily foods using LC-MS/MS. International Journal of Environmental Research and Public Health, v, 99, n. 6, p. 557-567, 2019. https://doi.org/10.1080/03067319.2019.1606218
ZHANG, X.; WANG, X.; LIU, Y.; FANG, K.; LIU, T. The Toxic Effects of Sulfoxaflor Induced in Earthworms (Eisenia fetida) under Effective Concentrations. International Journal of Environmental Research and Public Health, v. 17, n. 5, p. 1-14, 2020. https://doi.org/10.3390/ijerph17051740

ZHU, Y.; LOSO, M. R.; WATSON, G. B.; SPARKS, G. B.; ROGERS, R. B.; HUANG, J. X.; GERWICK, B. C.; BABCOCK, J. M.; KELLEY, D.; HEDGE, V. B.; NUGENT, B. M.; RENGA, J. M.; DENHOLM, I.; GORMAN, K.; DEBOER, G.; HASLER, J.; MEADE, T.; THOMAS, J. D. Discovery and Characterization of Sulfoxaflor, a Novel Insecticide Targeting Sap-Feeding Pests. Journal of Agricultural and Food Chemistry, v. 59, n. 7, p. 2950-2957, 2011. https://doi.org/10.1021/if102765x 